\title{
Corrigendum
}

\section{Programme National Nutrition Santé - guidelines score 2 (PNNS-GS2): development and validation of a diet quality score reflecting the 2017 French dietary guidelines - CORRIGENDUM}

Dan Chaltiel, Moufidath Adjibade, Valérie Deschamps, Mathilde Touvier, Serge Hercberg, Chantal Julia and Emmanuelle Kesse-Guyot

(First published online 25 July 2019)

DOI: https://doi.org/10.1017/S0007114519001181

Original text

Table 1 - PNNS-GS2: Components and scoring

\begin{tabular}{|c|c|c|c|}
\hline Dietary components & Recommendation & Criteria $^{a}$ & Score \\
\hline \multirow[t]{4}{*}{$\begin{array}{l}\text { Fruits and vegetables } \\
\text { (weight=3) }\end{array}$} & $\begin{array}{l}\text { At least } 5 \text { servings/day, with } 1 \text { max as juice } \\
\text { and } 1 \text { max as dried }\end{array}$ & $\begin{array}{l}{[0-3.5[} \\
{[3.5-5[} \\
{[5-7.5[} \\
\geq 7.5\end{array}$ & $\begin{array}{l}0 \\
0.5 \\
1 \\
2\end{array}$ \\
\hline & Prefer organic fruits & $\begin{array}{l}\text { Most of the time } \\
\text { Occasionally } \\
\text { Never }\end{array}$ & $\begin{array}{l}0.5 \\
0.25 \\
0\end{array}$ \\
\hline & \multirow[t]{2}{*}{ Prefer organic vegetables } & Most of the time & 0.5 \\
\hline & & $\begin{array}{l}\text { Occasionally } \\
\text { Never }\end{array}$ & $\begin{array}{l}0.25 \\
0\end{array}$ \\
\hline \multirow{2}{*}{$\begin{array}{l}\text { Nuts } \\
\text { (weight=1) }\end{array}$} & \multirow{2}{*}{ A handful/day } & 0 & 0 \\
\hline & & $\begin{array}{l}] 0-0.5[ \\
{[0.5-1.5[} \\
\geq 1.5\end{array}$ & $\begin{array}{l}0.5 \\
1 \\
0\end{array}$ \\
\hline \multirow[t]{5}{*}{$\begin{array}{l}\text { Legumes } \\
\text { (weight=1) }\end{array}$} & \multirow[t]{2}{*}{ At least 2 servings/week } & $\begin{array}{l}0 / \text { week } \\
] 0-2[/ \text { week }\end{array}$ & $\begin{array}{l}0 \\
0.5\end{array}$ \\
\hline & & $\geq 2$ /week & 1 \\
\hline & \multirow[t]{3}{*}{ Prefer organic legumes } & Most of time & 0.5 \\
\hline & & Occasionally & 0.25 \\
\hline & & Never & 0 \\
\hline \multirow{7}{*}{$\begin{array}{l}\text { Whole-grain food } \\
\text { (weight=2) }\end{array}$} & \multirow[t]{2}{*}{ Every day } & 0 & 0 \\
\hline & & $\begin{array}{l}] 0-1[ \\
{[1-2[}\end{array}$ & $\begin{array}{l}0.5 \\
1 \\
15\end{array}$ \\
\hline & \multirow{2}{*}{ Prefer organic bread } & Occasionally & 0.25 \\
\hline & & Never & 0 \\
\hline & \multirow{3}{*}{ Prefer organic grains } & Most of the time & 0.5 \\
\hline & & Occasionally & 0.25 \\
\hline & & Never & 0 \\
\hline \multirow{4}{*}{$\begin{array}{l}\text { Milk and dairy products } \\
\text { (weight=1) }\end{array}$} & \multirow[t]{4}{*}{2 servings/day } & {$[0-0.5[$} & 0 \\
\hline & & {$[0.5-1.5[$} & 0.5 \\
\hline & & {$[1.5-2.5[$} & 1 \\
\hline & & $\geq 2.5$ & 0 \\
\hline
\end{tabular}


Table 1 (Continued)

\begin{tabular}{|c|c|c|c|}
\hline Dietary components & Recommendation & Criteria $^{a}$ & Score \\
\hline $\begin{array}{l}\text { Red meat } \\
\text { (weight=2) }\end{array}$ & Limit consumption & $\begin{array}{l}{[0-500[\mathrm{~g} / \text { week }} \\
{[500-750[\mathrm{~g} / \text { week }} \\
\geq 750 \mathrm{~g} / \text { week }\end{array}$ & $\begin{array}{r}0 \\
-1 \\
-2\end{array}$ \\
\hline $\begin{array}{l}\text { Processed meat } \\
\text { (weight=3) }\end{array}$ & Limit consumption & $\begin{array}{l}{[0-150[\mathrm{~g} / \text { week }} \\
{[150-300[\mathrm{~g} / \text { week }} \\
\geq 300 \mathrm{~g} / \text { week }\end{array}$ & $\begin{array}{r}0 \\
-1 \\
-2\end{array}$ \\
\hline & Prefer white ham over other processed meat ${ }^{b}$ & $\begin{array}{l}\text { Ratio }<50 \% \\
\text { Ratio } \geq 50 \%\end{array}$ & $\begin{array}{l}0 \\
0.5\end{array}$ \\
\hline $\begin{array}{l}\text { Fish and seafood } \\
\text { (weight=2) }\end{array}$ & 2 servings/week & $\begin{array}{l}{[0-1.5 \text { [servings/week }} \\
{[1.5-2.5[\text { servings/week }} \\
{[2.5-3.5[\text { servings/week }} \\
\geq 3.5 \text { servings/week }\end{array}$ & $\begin{array}{l}0 \\
1 \\
0.5 \\
0\end{array}$ \\
\hline & Fatty fish 1 serving/week & $\begin{array}{l}{[0-0.5[\text { servings/week }} \\
{[0.5-1.5[\text { servings/week }} \\
\geq 1.5 \text { servings/week }\end{array}$ & $\begin{array}{l}0 \\
1 \\
0\end{array}$ \\
\hline $\begin{array}{l}\text { Added fat } \\
\text { (weight=2) }\end{array}$ & Avoid overeating & $\begin{array}{l}>16 \% \text { of EIWA }{ }^{c} \\
\leq 16 \% \text { of EIWA }\end{array}$ & $\begin{array}{l}0 \\
1.5\end{array}$ \\
\hline & Prefer ALA-rich and olive oil over other oils & $\begin{array}{l}\text { Ratio }<50 \% \\
\text { Ratio } \geq 50 \%\end{array}$ & $\begin{array}{l}0 \\
1\end{array}$ \\
\hline & Prefer vegetal fat over animal fat & $\begin{array}{l}\text { Ratio }>50 \% \\
\text { Ratio } \leq 50 \%\end{array}$ & $\begin{array}{l}0 \\
1\end{array}$ \\
\hline $\begin{array}{l}\text { Sugary foods } \\
\text { (weight=3) }\end{array}$ & Limit consumption & $\begin{array}{l}<10 \% \text { of EIWA } \\
{[10-15[\% \text { of EIWA }} \\
\geq 15 \% \text { of EIWA }\end{array}$ & $\begin{array}{r}0 \\
-1 \\
-2\end{array}$ \\
\hline $\begin{array}{l}\text { Sweet-tasting beverages }{ }^{d} \\
\text { (weight=3) }\end{array}$ & Limit consumption & $\begin{array}{l}0 \mathrm{~mL} / \text { day } \\
] 0-250[\mathrm{~mL} / \text { day } \\
{[250-750[\mathrm{~mL} / \text { day }} \\
\geq 750 \mathrm{~mL} \mathrm{~mL} / \text { day }\end{array}$ & $\begin{array}{l}0 \\
-0.5 \\
-1 \\
-2\end{array}$ \\
\hline $\begin{array}{l}\text { Alcoholic beverages } \\
\text { (weight=3) }\end{array}$ & Limit consumption & $\begin{array}{l}0 \mathrm{~g} / \text { week } \\
\text { ]0 - 100] g/week } \\
\text { ]100 - 200] g/week } \\
>200 \mathrm{~g} / \text { week }\end{array}$ & $\begin{array}{l}0.5 \\
0 \\
-1 \\
-2\end{array}$ \\
\hline $\begin{array}{l}\text { Salt } \\
\text { (weight=3) }\end{array}$ & Limit consumption & $\begin{array}{l}\leq 6 \text { g/day } \\
] 6-8] \mathrm{g} / \text { day } \\
\text { ] } 8-10] \mathrm{g} / \text { day } \\
\text { ]10 - 12] g/day } \\
>12 \mathrm{~g} / \text { day }\end{array}$ & $\begin{array}{l}1 \\
0 \\
-0.5 \\
-1 \\
-2\end{array}$ \\
\hline
\end{tabular}

Principal recommendations are written in bold.

a Servings per day unless otherwise stated

${ }^{b}$ Conditional : the 0.5 bonus point only occurs if total processed meat consumption is more than $150 \mathrm{~g} /$ week

${ }^{c}$ EIWA $=$ energy intake without alcohol

d Sweet-tasting beverages are specifically sugary beverages, artificially sweetened beverages and fruit juices

Table 1 - PNNS-GS2: Components and scoring

\begin{tabular}{|c|c|c|c|}
\hline Dietary components & Recommendation & Criteria $^{a}$ & Score \\
\hline \multirow[t]{7}{*}{$\begin{array}{l}\text { Fruits and vegetables } \\
\text { (weight=3) }\end{array}$} & $\begin{array}{l}\text { At least } 5 \text { servings/day, with } 1 \text { max as juice } \\
\text { and } 1 \text { max as dried }\end{array}$ & $\begin{array}{l}{[0-3.5[} \\
{[3.5-5[} \\
{[5-7.5[} \\
\geq 7.5\end{array}$ & $\begin{array}{l}0 \\
0.5 \\
1 \\
2\end{array}$ \\
\hline & \multirow[t]{3}{*}{ Prefer organic fruits } & Most of the time & 0.5 \\
\hline & & Occasionally & 0.25 \\
\hline & & Never & 0 \\
\hline & \multirow[t]{3}{*}{ Prefer organic vegetables } & Most of the time & 0.5 \\
\hline & & Occasionally & 0.25 \\
\hline & & Never & 0 \\
\hline \multirow{4}{*}{$\begin{array}{l}\text { Nuts } \\
\text { (weight=1) }\end{array}$} & \multirow[t]{4}{*}{ A handful/day } & 0 & 0 \\
\hline & & ] $0-0.5[$ & 0.5 \\
\hline & & {$[0.5-1.5[$} & 1 \\
\hline & & $\geq 1.5$ & 0 \\
\hline
\end{tabular}


Table 1 (Continued)

\begin{tabular}{|c|c|c|c|}
\hline Dietary components & Recommendation & Criteria $^{a}$ & Score \\
\hline \multirow[t]{4}{*}{$\begin{array}{l}\text { Legumes } \\
\text { (weight=1) }\end{array}$} & At least 2 servings/week & $\begin{array}{l}0 / \text { week } \\
] 0-2[\text { /week } \\
\geq 2 \text { /week }\end{array}$ & $\begin{array}{l}0 \\
0.5 \\
1\end{array}$ \\
\hline & \multirow[t]{3}{*}{ Prefer organic legumes } & Most of time & 0.5 \\
\hline & & Occasionally & 0.25 \\
\hline & & Never & 0 \\
\hline \multirow{10}{*}{$\begin{array}{l}\text { Whole-grain food } \\
\text { (weight=2) }\end{array}$} & \multirow[t]{4}{*}{ Every day } & 0 & 0 \\
\hline & & ] $0-1[$ & 0.5 \\
\hline & & {$[1-2[$} & 1 \\
\hline & & $\geq 2$ & 1.5 \\
\hline & \multirow[t]{3}{*}{ Prefer organic bread } & Most of the time & 0.5 \\
\hline & & Occasionally & 0.25 \\
\hline & & Never & 0 \\
\hline & \multirow[t]{3}{*}{ Prefer organic grains } & Most of the time & 0.5 \\
\hline & & Occasionally & 0.25 \\
\hline & & Never & 0 \\
\hline \multirow{4}{*}{$\begin{array}{l}\text { Milk and dairy products } \\
\text { (weight=1) }\end{array}$} & \multirow[t]{4}{*}{2 servings/day } & {$[0-0.5[$} & 0 \\
\hline & & {$[0.5-1.5[$} & 0.5 \\
\hline & & {$[1.5-2.5[$} & 1 \\
\hline & & $\geq 2.5$ & 0 \\
\hline \multirow{3}{*}{$\begin{array}{l}\text { Red meat } \\
\text { (weight=2) }\end{array}$} & \multirow[t]{3}{*}{ Limit consumption } & {$[0-500[\mathrm{~g} /$ week } & 0 \\
\hline & & {$[500-750[\mathrm{~g} /$ week } & -1 \\
\hline & & $\geq 750 \mathrm{~g} /$ week & -2 \\
\hline \multirow{5}{*}{$\begin{array}{l}\text { Processed meat } \\
\text { (weight=3) }\end{array}$} & \multirow[t]{3}{*}{ Limit consumption } & {$[0-150[\mathrm{~g} /$ week } & 0 \\
\hline & & {$[150-300[\mathrm{~g} /$ week } & -1 \\
\hline & & $\geq 300 \mathrm{~g} /$ week & -2 \\
\hline & \multirow{2}{*}{ Prefer white ham over other processed meat ${ }^{\mathrm{b}}$} & Ratio $<50 \%$ & 0 \\
\hline & & Ratio $\geq 50 \%$ & 0.5 \\
\hline \multirow{7}{*}{$\begin{array}{l}\text { Fish and seafood } \\
\text { (weight=2) }\end{array}$} & \multirow[t]{4}{*}{2 servings/week } & {$[0-1.5[$ servings/week } & 0 \\
\hline & & {$[1.5-2.5[$ servings/week } & 1 \\
\hline & & {$[2.5-3.5[$ servings/week } & 0.5 \\
\hline & & $\geq 3.5$ servings/week & 0 \\
\hline & \multirow[t]{3}{*}{ Fatty fish 1 serving/week } & {$[0-0.5[$ servings/week } & 0 \\
\hline & & {$[0.5-1.5[$ servings/week } & 1 \\
\hline & & $\geq 1.5$ servings/week & 0 \\
\hline \multirow{6}{*}{$\begin{array}{l}\text { Added fat } \\
\text { (weight=2) }\end{array}$} & \multirow[t]{2}{*}{ Avoid overeating } & $>16 \%$ of $\mathrm{EIWA}^{\mathrm{C}}$ & 0 \\
\hline & & $\leq 16 \%$ of EIWA & 1.5 \\
\hline & Prefer ALA-rich and olive oil over other oils & Ratio $<50 \%$ & 0 \\
\hline & & Ratio $\geq 50 \%$ & 1 \\
\hline & Prefer vegetal fat over animal fat & Ratio $<50 \%$ & 0 \\
\hline & & Ratio $\geq 50 \%$ & 1 \\
\hline Sugary foods & Limit consumption & $<10 \%$ of EIWA & 0 \\
\hline (weight=3) & & {$[10-15[\%$ of ElWA } & -1 \\
\hline & & $\geq 15 \%$ of EIWA & -2 \\
\hline Sweet-tasting beverages ${ }^{d}$ & Limit consumption & $0 \mathrm{~mL} /$ day & 0 \\
\hline$($ weight $=3)$ & & ] $0-250[\mathrm{~mL} / \mathrm{day}$ & -0.5 \\
\hline & & {$[250-750[\mathrm{~mL} /$ day } & -1 \\
\hline & & $\geq 750 \mathrm{~mL} \mathrm{~mL} /$ day & -2 \\
\hline Alcoholic beverages & Limit consumption & $0 \mathrm{~g} /$ week & 0.5 \\
\hline (weight=3) & & ]0 - 100] g/week & 0 \\
\hline & & ]100 - 150] g/week & -1 \\
\hline & & ]150 - 200] g/week & -1.5 \\
\hline & & $>200 \mathrm{~g} / \mathrm{week}$ & -2 \\
\hline Salt & Limit consumption & $<6$ g/day & 1 \\
\hline (weight=3) & & {$[6-8[\mathrm{~g} / \mathrm{day}$} & 0 \\
\hline & & {$[8-10[\mathrm{~g} /$ day } & -0.5 \\
\hline & & {$[10-12[\mathrm{~g} /$ day } & -1 \\
\hline & & $\geq 12 \mathrm{~g} /$ day & -2 \\
\hline
\end{tabular}

Principal recommendations are written in bold.

a Servings per day unless otherwise stated

${ }^{b}$ Conditional : the 0.5 bonus point only occurs if total processed meat consumption is more than $150 \mathrm{~g} / \mathrm{week}$

${ }^{c}$ EIWA = energy intake without alcohol

${ }^{d}$ Sweet-tasting beverages are specifically sugary beverages, artificially sweetened beverages and fruit juices 